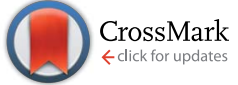

Cite this: RSC Adv., 2017, 7, 8106

Received 28th October 2016 Accepted 16th January 2017

DOI: $10.1039 / c 6 r a 25980 d$

www.rsc.org/advances

\section{Two magnon scattering and anti-damping behavior in a two-dimensional epitaxial $\mathrm{TiN} / \mathrm{Py}\left(t_{\mathrm{Py}}\right) / \beta-\mathrm{Ta}\left(t_{\mathrm{Ta}}\right)$ system $\uparrow$}

\begin{abstract}
Nilamani Behera, ${ }^{a}$ Ankit Kumar, ${ }^{\text {ab }}$ Sujeet Chaudhary ${ }^{\star a}$ and Dinesh K. Pandya ${ }^{a}$
The prime requirements for the spin transfer torque based ferromagnetic (FM)/nonmagnetic (NM) bilayer spin devices are (i) the absence of two-magnon scattering (TMS) noise, (ii) minimum energy dissipation and (iii) fast switching. To realize these objectives we have studied the thickness, Py (permalloy) thicknesses $\left(t_{P y}\right)$ and $\beta$-Ta thicknesses $\left(t_{T a}\right)$, dependent magnetization dynamics behaviour of the epitaxial Py $\left(t_{P y}=3-20 \mathrm{~nm}\right) / \beta-T a\left(t_{T a}=1.5-15 \mathrm{~nm}\right)$ system. The $t_{P y}$ dependence of TMS in epitaxial Py nanolayers $\left(t_{\mathrm{Py}}=3-20 \mathrm{~nm}\right)$ grown on a $\mathrm{Si}(400) / \mathrm{TiN}(200)(8 \mathrm{~nm})$ substrate is explored in terms of uniform and non-uniform magnetization precession regimes by employing ferromagnetic resonance field $\left(H_{r}\right)$, linewidth $(\Delta H)$, and Gilbert damping constant $(\alpha)$ behaviour. It is found that in Py, $t_{\mathrm{Py}}<10 \mathrm{~nm}$, layers TMS is dominating due to non-uniform precession of the magnetization. However in $P y, t_{P y} \geq 10 \mathrm{~nm}$, layers the uniform magnetization precession dominates, therefore Py layers, $t_{P y} \geq 10 \mathrm{~nm}$, are almost free from TMS. Furthermore, a nearly TMS free $12 \mathrm{~nm}$ epitaxial Py(200) layer is capped with $\beta$-Ta $\left(t_{\mathrm{Ta}}=1.5-15 \mathrm{~nm}\right.$ ) layers to explore the $t_{T a}$ dependent magnetization precession of epitaxial Py $(12 \mathrm{~nm})$ in terms of change in effective Gilbert damping constant $\left(\alpha_{\text {eff }}\right)$. An anomalous decrease in $\alpha_{\text {eff }}$ from 0.0087 at $t_{\text {Ta }}=0$ to a minimum value of 0.0077 at $t_{\mathrm{Ta}}=6 \mathrm{~nm}$, and its subsequent increase for $t_{\mathrm{Ta}}>6 \mathrm{~nm}$ are observed in the epitaxial Py $(12 \mathrm{~nm}) / \beta-\mathrm{Ta}\left(t_{\mathrm{Ta}}\right)$ system. Therefore the $\mathrm{Si}(400) / \mathrm{TiN}(200)(8 \mathrm{~nm}) / \mathrm{Py}(200)(12 \mathrm{~nm}) / \beta-\mathrm{Ta}(200)$ $(6 \mathrm{~nm})$ epitaxial system with nearly uniform magnetic precession and minimum effective Gilbert damping is suitable for low energy loss and ultrafast switching applications in spin transfer torque devices.
\end{abstract}

\section{Introduction}

Employing spin current without charge current is of great interest in the development of spin transfer torque (STT) devices. ${ }^{1}$ A bilayer consisting of ferromagnetic (FM)/nonmagnetic (NM) layers is one of the most reliable material architectures to fundamentally investigate the STT mechanism and its role in devices. ${ }^{2-7}$ Owing to the presence of structural inversion asymmetry among the two dissimilar materials at the FM/NM interface, an important physical phenomenon known as the Rasbha effect results when a charge current flows through the bilayer, wherein the spin-orbit Hamiltonian breaks the degeneracy in the electron spin states at the interface and creates a non equilibrium spin accumulation. ${ }^{8}$ This nonequilibrium spin accumulation acts like a spin-orbit torque (SOT) on the magnetization precession of the FM layer. ${ }^{8,9}$ Thus, the Rashba effect gives rise to a spin-orbit torque (SOT) which

${ }^{a}$ Thin Film Laboratory, Department of Physics, Indian Institute of Technology Delhi, New Delhi 110016, India

${ }^{b}$ Department of Engineering Sciences, Uppsala University, Box 534, 75121 Uppsala, Sweden.E-mail:sujeetc@physics.iitd.ac.in

$\dagger$ Electronic supplementary information (ESI) available. See DOI: 10.1039/c6ra25980d plays a critical role in the FM/NM bilayer systems by enhancing the efficiency of STT in potential device applications. ${ }^{8,9}$ The SOT arises from the non-equilibrium spin accumulation at interface in presence of strong spin-orbit interaction (SOI) of NM layer in FM/NM bilayer system. ${ }^{10}$ The spin-pumping induced spin accumulation creates a non-equilibrium spin density in the adjacent NM layer near the FM/NM interface. Thus in FM/NM bilayer system two types of spin-orbit torque exist, (i) interfacial spin-orbit coupling (ISOC) torque due to Rashba effect and (ii) bulk spin-orbit coupling (BSOC) torque due to spin Hall effect or spin accumulation in NM layer. ${ }^{\mathbf{1 1}, \mathbf{1 2}}$ Therefore the net torque acting on the FM layer in FM/NM bi-layer system can be represented as, $\vec{T}=\left(T_{\mathrm{ISOC}}+T_{\mathrm{BSOC}}\right)_{\perp}[\hat{y} \times \hat{m}]+\left(T_{\mathrm{ISOC}}+T_{\mathrm{BSOC}}\right)_{\|}[\hat{m}$ $\times \hat{y} \times \hat{m}]$, where $\hat{y}$ is the spin polarization direction and $\hat{m}$ is magnetization direction. Here $\left(T_{\mathrm{ISOC}}+T_{\mathrm{BSOC}}\right)_{\perp}$ is the field-like (or out of plane) toque, and $\left(T_{\mathrm{ISOC}}+T_{\mathrm{BSOC}}\right)_{\|}$is the antidamping (or in-plane) torque, ${ }^{\mathbf{1 1}, \mathbf{1 2}}$ both having contributions from interfacial and bulk spin-orbit couplings. The field-like toque is exchange-coupled torque, and apart from its Rashba origin it also can have origin in a non-adiabatic spin transfer torque. The field-like torque enhances the precession of the magnetization of the FM layer at high current density. ${ }^{13}$ On the other hand, anti-damping torque is predominantly Slonczewski Berger spin-transfer torque, ${ }^{\mathbf{1 4}}$ and it can also have contribution 
from spin flip reflection at the interface..$^{\mathbf{1 0 - 1 3 , 1 5}}$ Thus, ISOC and BSOC induced torque play crucial role in realizing low power magnetization switching, ${ }^{5}$ high speed domain wall motion, ${ }^{\mathbf{1 6 - 1 8}}$ tunable nano oscillators, ${ }^{4,19}$ etc. They also help in reducing the writing energy and improving device life time. ${ }^{\mathbf{1 8 , 1 9}}$

However it has been recently reported that Rashba SOT occurs in FM/NM bilayer system because of non-equilibrium spin accumulation nearer to the interface. These nonequilibrium spins accumulate at the interface either by SHE phenomena with application of charge current in $\mathrm{NM}_{\text {layer }}{ }^{\mathbf{1 9}}$ or from the spin pumping from FM layer in the absence of any external dc current in FM/NM bilayer system or inversion symmetry breaking at the interface..$^{\mathbf{2 0 - 2 6}}$ These accumulated spins do not dissipate their angular momentum in the NM layer within the thickness limits of spin diffusion length $\left(\lambda_{\mathrm{SD}}\right) .^{\mathbf{1 4 , 2 3 , 2 4 , 2 7}}$ Thus the choice of NM layer for the Rashba and BSOC SOT mechanisms is significant for efficient pure spin current based new generation spintronic devices. Since $\beta$-Ta has spin accumulation property along with high - ve spin Hall angle ( $\sim 2$ times that in $\mathrm{Pt}$ ) and high SOI value (comparable to that in Pt).$^{\mathbf{6} 21} \mathrm{It}$ provides an attractive alternative to Pt as heavy metal NM layer for SOT phenomena. Study on Py/ $\beta$-Ta bilayers with polycrystalline Py reported that as the $\beta$-Ta layer thickness is varied a lowering of damping is observed. ${ }^{26}$ As already stated the Rashba SOT phenomenon can be studied efficiently when the FM layer is undergoing uniform magnetization precession. ${ }^{\mathbf{1 3 , 2 8 - 3 0}}$ It is therefore imperative to focus and improve upon the quality as well as tune the thickness of FM layer such that the uniform precession of magnetization (known as Gilbert type of relaxation) should exist without any interruption arising due to extrinsic effects that lead to non-uniform magnetizationprecession (non-Gilbert types of relaxation). ${ }^{31,32}$ These extrinsic effects could be either the presence of surface anisotropy field, or magnetic inhomogeneity, or dipolar coupling field between the magnetic moments, all of which make the magnetization precession of FM layer increasingly non-uniform in nature resulting in two magnon scattering (TMS) phenomena. ${ }^{31,32}$ In the TMS process, the uniform magnetization precession at a particular frequency is annihilated and another spin wave magnon of the same frequency (akin to discrete quantum modes) is created which leads to the enhancement in FMR linewidth and hence in the damping of magnetization precession..$^{31-33}$ To avoid such undesirable features that add to the Gilbert damping constant, it seems necessary to concentrate on appropriate thickness of epitaxial Py FM layer such that the uniform precession of magnetization should exist. Thus, the critical film thickness regime needs to be carefully identified for minimization of extrinsic TMS contribution. In this work, we therefore have focused on epitaxial permalloy (Py) FM layer of thickness up to $20 \mathrm{~nm}$, with the use of $\mathrm{Si}(100)$ wafer/8 $\mathrm{nm} \operatorname{TiN}(200)$ substrate instead of using $\mathrm{Si} / \mathrm{SiO}_{2}$ substrate (where $\mathrm{SiO}_{2}$ is a native oxide layer). Here epi-TiN acts as buffer layer useful for minimizing the lattice mismatch problem between Si substrate and Py layer., ${ }^{34,35}$ TiN is a material which has high conductivity and low spin-orbit coupling strength. The spin diffusion length in TiN is $43 \mathrm{~nm} .^{36}$ The growth of $\operatorname{TiN}(200)$ layer occurs by domain epitaxy process. ${ }^{34}$ Then we optimize the particular thickness regime of Py layer so that the TMS contribution is minimum. After that we studied the effect of $\beta$-Ta layer thickness (1.5-15 $\mathrm{nm})$ on the magnetization precession of Py layer in $\operatorname{TiN}(200) / \mathrm{Py}(200) / \beta$-Ta system. It is not only technologically important but also of fundamental interest to explore the role of $\beta$-Ta layer on the SOT mechanism. We discuss the detailed results related with the observed antidamping behavior of TiN/Py/ $\beta$-Ta multilayers in terms of the changes in the effective Gilbert damping constant of uniformly magnetized epi-Py layer of appropriate thickness as a function of the $\beta$-Ta layer film thickness with naturally formed $\beta-\mathrm{Ta}_{2} \mathrm{O}_{5}$ oxide interface $\left(\mathrm{Ta}_{2} \mathrm{O}_{5}\right.$ layer $(\sim 2 \mathrm{~nm})$ is formed naturally after the exposure to ambient). The results are organized in three different sections; (A) the growth of epitaxial Py $(3-20 \mathrm{~nm})$ on $8 \mathrm{~nm}$ epitaxial TiN buffer, (B) study of the magnetization dynamics in these epitaxial Py samples to investigate the thickness dependent TMS, and (C) study of the $\beta$-Ta layer thickness (1.5-15 nm) dependent effective Gilbert damping constant in $\operatorname{TiN}(200) / \mathrm{Py}(200) / \beta$-Ta bilayers.

\section{Experimental}

Two different sets of samples were prepared for the present studies. In the first set, Py $\left(\mathrm{Ni}_{81} \mathrm{Fe}_{19}\right)$ thin films of different thicknesses 3-20 nm were grown on epitaxial TiN buffered $\mathrm{Si}$ substrates by pulsed DC magnetron sputtering using 99.99\% pure Py target room temperature. While the base pressure of sputtering chamber was $\sim 2 \times 10^{-7}$ Torr, the sputtering was performed in the Ar environment maintained at a working pressure of $3.2 \times 10^{-3}$ Torr. Prior to deposition, the Si substrates were treated to remove the native $\mathrm{SiO}_{2}$ layer by dipping it into $5 \% \mathrm{HF}$ solution for $1 \mathrm{~min}$. Subsequently, the epitaxial TiN films were grown on these $\mathrm{Si}$ substrates at $750{ }^{\circ} \mathrm{C}$ in presence of $\mathrm{N}_{2}$ partial pressure of $5 \times 10^{-5}$ Torr. $^{35}$ In the second set, $\beta$-Ta layers of different thicknesses varying from 1.5 to $15 \mathrm{~nm}$ were grown on the epitaxial $\operatorname{Si}(100) / \operatorname{TiN}(8 \mathrm{~nm}) / \operatorname{Py}(12 \mathrm{~nm})$. The growth of $\beta$ $\mathrm{Ta}(200)$ oriented films were made by employing pulsed dc magnetron sputtering using 99.99\% pure Ta target at room temperature. During the growth the Ar gas pressure was maintained at $3.2 \times 10^{-3}$ Torr. The films were grown at $150 \mathrm{~W}$ $\left(\sim 0.2 \mathrm{~nm} \mathrm{~s}^{-1}\right)$ sputtering power. The measured values of resistivity of these films, lying in the range of 180-190 $\mu \Omega \mathrm{cm}$, matched excellently well with the reported value of $\beta$-phase Ta in literature. ${ }^{5,11}$ Substrate surface and the growing films were monitored by the high pressure reflection high energy electron diffraction (RHEED) of STAIB Instruments, and RHEED patterns were analyzed using KSA-400 software. Before the deposition, the substrates were heated at $750{ }^{\circ} \mathrm{C}$. The clear RHEED patterns observed after the heat treatment ensured the cleanliness of the substrate surface. The crystal structure analyses were performed using X'Pert-Pro X-ray Diffractometer (XRD) with Cu- $\mathrm{K}_{\alpha}(1.54 \AA$ ) source. The thicknesses of individual layers and interface roughness $(\sim 0.4 \mathrm{~nm})$ were accurately determined from the XRR measurements. X-ray photoelectron spectroscopic (XPS) spectra were recorded using SPECS make system which uses Al-K $\mathrm{K}_{\alpha}(1486.6$ $\mathrm{eV}$ ) source and hemispherical energy analyzer (pass energy of $40 \mathrm{eV}$ with a resolution of $\sim 0.3 \mathrm{eV}$ ) to probe the surface of the $\mathrm{Py} / \beta$-Ta bilayers. The in-plane magnetization measurements of 
$\mathrm{Py} / \beta$-Ta thin films were measured by using Vibrating sample magnetometer option of Physical Property Measurement System (Model Evercool II, Quantum Design Inc.). The resonance field $H_{\mathrm{r}}$ and line width $\Delta H$ were measured by using broadband lock-in amplifier (SR-830) based ferromagnetic resonance (LIA-FMR) technique with the help of a 8719ES vector network analyzer (VNA) in the in-plane magnetic field configuration (along the easy magnetization direction) employing a coplanar wave guide (CPW). The dc-magnetic field was modulated with a $211.5 \mathrm{~Hz}$ acfield of 1.3 Oe strength using a pair of Helmholtz coils.

\section{Results and discussion}

\section{A. RHEED and Structural analyses of Py on epitaxial TiN}

The RHEED pattern recorded on bare Si(100) along the [001] direction is shown in Fig. 1(a). The sharp streaky pattern with Kikuchi lines clearly confirms the clean and stable $(2 \times 1)$ reconstructed Si surface. Fig. 1(b) shows the RHEED pattern recorded along [001] direction on $\mathrm{TiN}(200)$ film grown on $(2 \times$ 1) reconstructed $\operatorname{Si}(100)$ substrate. Regardless of the $\sim 22.4 \%$ lattice misfit of $\mathrm{TiN}$ with $\mathrm{Si}(100)$, presence of prolonged streaks confirm the epitaxial growth of $\operatorname{TiN}(200)$ on $\mathrm{Si}(100)$ which is also confirmed by XRD and will be discussed in the next section. This has been possible by domain epitaxy ${ }^{34}$ because of a specific orientation relationship in which 5 unit cells of $\operatorname{TiN}(a=4.24 \AA)$ match with 4 unit cells of $\mathrm{Si}(a=5.43 \AA)$, which reduces the overall mismatch to $2.4 \%$ and hence $\mathrm{Si}(400) / / \mathrm{TiN}(200) ; \mathrm{Si}[001] / /$ TiN[001] epitaxial growth occurs. ${ }^{35}$ The Py layers of different
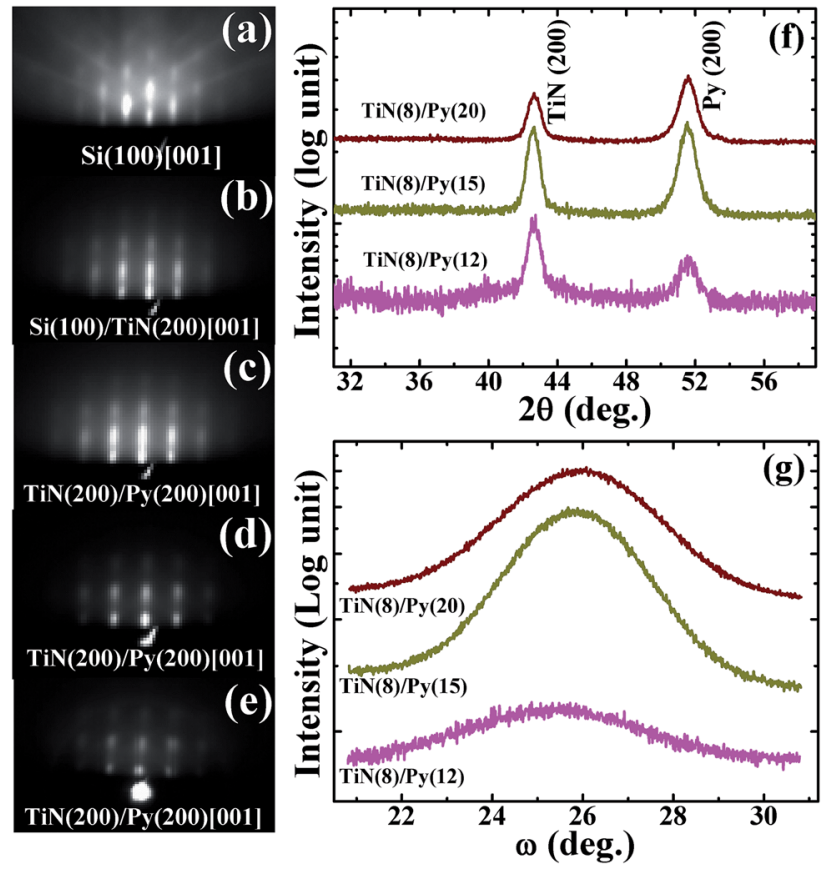

Fig. 1 The RHEED patterns along [001] of (a) cleaned Si(100) substrate, (b) TiN 8 nm, (c) Py 20 nm, (d) Py 12 nm, and (e) Py $7 \mathrm{~nm}$ samples. (f) Typical $\theta-2 \theta$ XRD pattern of epitaxial TiN $(8 \mathrm{~nm}) / \mathrm{Py}\left(t_{\mathrm{Py}} \mathrm{nm}\right)$ films on $\mathrm{Si}(100)$ substrate showing high crystalline quality, single phase Py with (200) orientation. (g) shows the $\omega$-scan XRD of 20, 15, and $12 \mathrm{~nm}$ Py samples. thicknesses in 3-20 nm range were deposited on TiN buffered Si(100). 2-D epitaxial growth was seen to occur for Py layer thicknesses $\geq 12 \mathrm{~nm}$. Fig. 1(c)-(e) show the RHEED patterns recorded along [001] direction for 20, 12, $7 \mathrm{~nm}$ Py layer samples, respectively. The evolution of elongated and sharp streaks with the increase in the Py layer thickness confirms the improvement in epitaxial quality of the Py layers. Similar RHEED patterns are also observed along [010] direction (not shown here for brevity). Thus, the RHEED patterns along [001] and [010] clearly confirm the in-plane epitaxial $\operatorname{TiN}(200) / / \mathrm{Py}(200) ; \operatorname{TiN}[001] / / \mathrm{Py}[001]$ orientation relationship of these samples.

To further investigate the crystallographic orientation of Py $\left(t_{\mathrm{Py}} \mathrm{nm}\right)$ thin films, where $t_{\mathrm{Py}}$ corresponds to thickness of Py layer, the $\theta-2 \theta$ scans and rocking $\omega$-scans were also recorded. The $\theta-2 \theta$ scans of the bilayer TiN/Py samples are shown in Fig. 1(f). The observed diffraction peak from the (200) planes of Py layer on TiN(200) together with the RHEED analysis further confirmed the epitaxial $\mathrm{Si}(400) / \mathrm{TiN}(200) / \mathrm{Py}(200)$ orientation relationship of all the samples. As expected, these samples did not show any diffraction peak in glancing angle XRD measurements (data not shown for brevity). The $\omega$-scan XRD profiles (Fig. 1(g)) recorded on these samples clearly suggest that the increase in Py layer thickness leads to the enhancement in the crystalline quality. Also, the presence of relatively sharp and streaky RHEED patterns confirms the changeover of the epitaxial growth from three dimensional $\left(t_{\mathrm{Py}}<10 \mathrm{~nm}\right)$ to twodimensional $\left(t_{\mathrm{Py}} \geq 10 \mathrm{~nm}\right)$ as shown Fig. 1 in ESI file. $\dagger$

\section{B. Study of the magnetization dynamics of epitaxial Py thin films}

To explore the spin dynamic response in these samples, the FMR spectra were recorded by sweeping the external magnetic field from higher to lower values at different constant frequencies in the range of 5 to $10 \mathrm{GHz}$. The FMR measurements are performed on the epitaxial $\mathrm{Si} / \mathrm{TiN}(8 \mathrm{~nm}) / \mathrm{Py}\left(t_{\mathrm{Py}} \mathrm{nm}\right)$ system to identify the thickness regime of the Py layer for which the $\Delta H$ is minimum so as to have a tradeoff between film thickness and low extrinsic contributions. The details of measurement setup are given in ref. 26. The FMR absorption spectra were recorded on different samples of the $\mathrm{Si}(100) / \mathrm{TiN}(8$ $\mathrm{nm}) / \mathrm{Py}\left(t_{\mathrm{Py}} \mathrm{nm}\right)$ series, where $t_{\mathrm{Py}}$ ranges in 3-20 $\mathrm{nm}$. For clarity and conciseness, Fig. 2 shows the FMR absorption curves

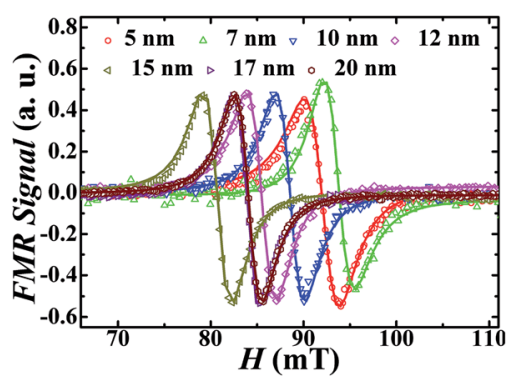

Fig. 2 The FMR absorption curves (experimental data in open symbols) of TiN $(8 \mathrm{~nm}) / \mathrm{Py}\left(t_{\mathrm{Py}} \mathrm{nm}\right)$ at $9 \mathrm{GHz}$, where $t_{\text {Py }}$ is the thickness of epitaxial Py layer over the range of $5-20 \mathrm{~nm}$. The solid lines represent the fitted curves. 
recorded at one of the frequencies $(9 \mathrm{GHz})$ for the different samples. Each of the FMR spectrum is fitted with sum of the derivatives of symmetric and antisymmetric Lorentzian functions, as shown by the solid lines in Fig. 2, to determine the values of in-plane $\Delta H$ and $H_{\mathrm{r}}$, using: ${ }^{6}$

$$
\text { Resonance signal } \approx A \frac{\partial\left(F_{\mathrm{S}}\left(H_{\text {ext. }}\right)\right)}{\partial H_{\text {ext. }}}+B \frac{\partial\left(F_{\mathrm{A}}\left(H_{\text {ext. }}\right)\right)}{\partial H_{\text {ext. }}}
$$

here, $F_{\mathrm{S}}\left(H_{\text {ext. }}\right)=\Delta H^{2} /\left\{\Delta H^{2}+\left(H_{\text {ext. }}-H_{\mathrm{r}}\right)^{2}\right\}$, and $F_{\mathrm{A}}\left(H_{\text {ext. }}\right)=$ $F_{\mathrm{S}}\left(H_{\text {ext. }}\right)\left\{\left(H_{\text {ext. }}-H_{\mathrm{r}}\right) / \Delta H\right\}$ are symmetric and antisymmetric Lorentzian functions, respectively, with constants $A$ and $B$ as their corresponding coefficients. When fitted using eqn (1), it is found that the observed FMR spectra are of completely symmetric Lorentzian shape, and the antisymmetric Lorentzian coefficients are found to be very small as compared to symmetric Lorentzian coefficient (by a factor of 100 or more). The frequency and thickness dependences of the observed line shape parameters $\left(H_{\mathrm{r}}\right.$ and $\left.\Delta H\right)$ are plotted in Fig. 3. To understand the observed variation of in-plane $H_{\mathrm{r}}$ with the resonance frequency $f$ for series of samples $\left(t_{\mathrm{NM}}: 3-20 \mathrm{~nm}\right)$ the observed line shape parameters are fitted by using the Kittel's eqn; ${ }^{37}$

$$
f=\frac{\gamma}{2 \pi}\left[H_{\mathrm{r}}\left(H_{\mathrm{r}}+4 \pi M_{\mathrm{eff}}\right)\right]^{\frac{1}{2}}
$$

where, $\gamma$ is the gyromagnetic ratio $\left(=1.856 \times 10^{11} \mathrm{~Hz} \mathrm{~T}^{-1}\right)$, $4 \pi M_{\text {eff }}$ is the effective magnetization field, which is given by:
$4 \pi M_{\text {eff }}=4 \pi M_{\mathrm{S}}+H_{\mathrm{S}}$. Here $H_{\mathrm{S}}$ is the surface anisotropy field $\left(=\frac{2 K_{\mathrm{S}}}{M_{\mathrm{S}} t_{\mathrm{Py}}}\right)$ which is induced by the surface and interfacial defects and also by the anisotropic orbital angular momentum, ${ }^{38} 4 \pi M_{\mathrm{S}}$ is the saturation magnetization of Py layer, and $K_{\mathrm{S}}$ is the surface anisotropy energy constant. The value of $4 \pi M_{\mathrm{s}}$, as determined from the fitting of $4 \pi M_{\mathrm{eff}} v s . t_{\mathrm{Py}}$ data using the expression $4 \pi M_{\mathrm{eff}}=4 \pi M_{\mathrm{s}}+H_{\mathrm{S}}\left(=\frac{2 K_{\mathrm{S}}}{M_{\mathrm{s}} t_{\mathrm{Py}}}\right)$, is found to be $1088( \pm 19) \mathrm{mT}$ (see Fig. ESI5(a)†). The $4 \pi M_{\mathrm{s}}$ value obtained from FMR measurements is consistent with the value of 1195 $( \pm 100) \mathrm{mT}$ obtained from VSM measurements. ${ }^{39}$ Here it is to be noted that one can't directly extract the $H_{\mathrm{S}}$ from the effective magnetization because $H_{\mathrm{S}}$ is also affected by shift in the $H_{\mathrm{r}}$ with respect to $t_{\mathrm{Py}}{ }^{40}$ Fig. 3(a) shows the variation of $H_{\mathrm{r}}$ with $f$ for different $\operatorname{Py}\left(t_{\mathrm{Py}}\right)$ films, and this observed variation is fitted to eqn (2). The fitted values of $4 \pi M_{\text {eff }}$ are found to vary from 772 $\mathrm{mT}$ to $1028 \mathrm{mT}$ as Py thickness increases from 3 to $20 \mathrm{~nm}$ (see Fig. ESI5a †). Fig. 3(b) shows a shift in the resonance field $H_{\mathrm{r}}$ on increasing $t_{\mathrm{Py}}$ corresponding to different constant resonance frequencies. This shift can be linked with the nature of resonance mode associated with the samples. To explain this $t_{\mathrm{Py}}$ dependence of $H_{\mathrm{r}}$, we utilized the following expression, ${ }^{37}$

$$
f=\frac{\gamma}{2 \pi}\left[H_{\mathrm{r}}\left(H_{\mathrm{r}}+4 \pi M_{\mathrm{eff}}\right)\right]^{\frac{1}{2}}-r H_{\mathrm{S}}^{2}
$$
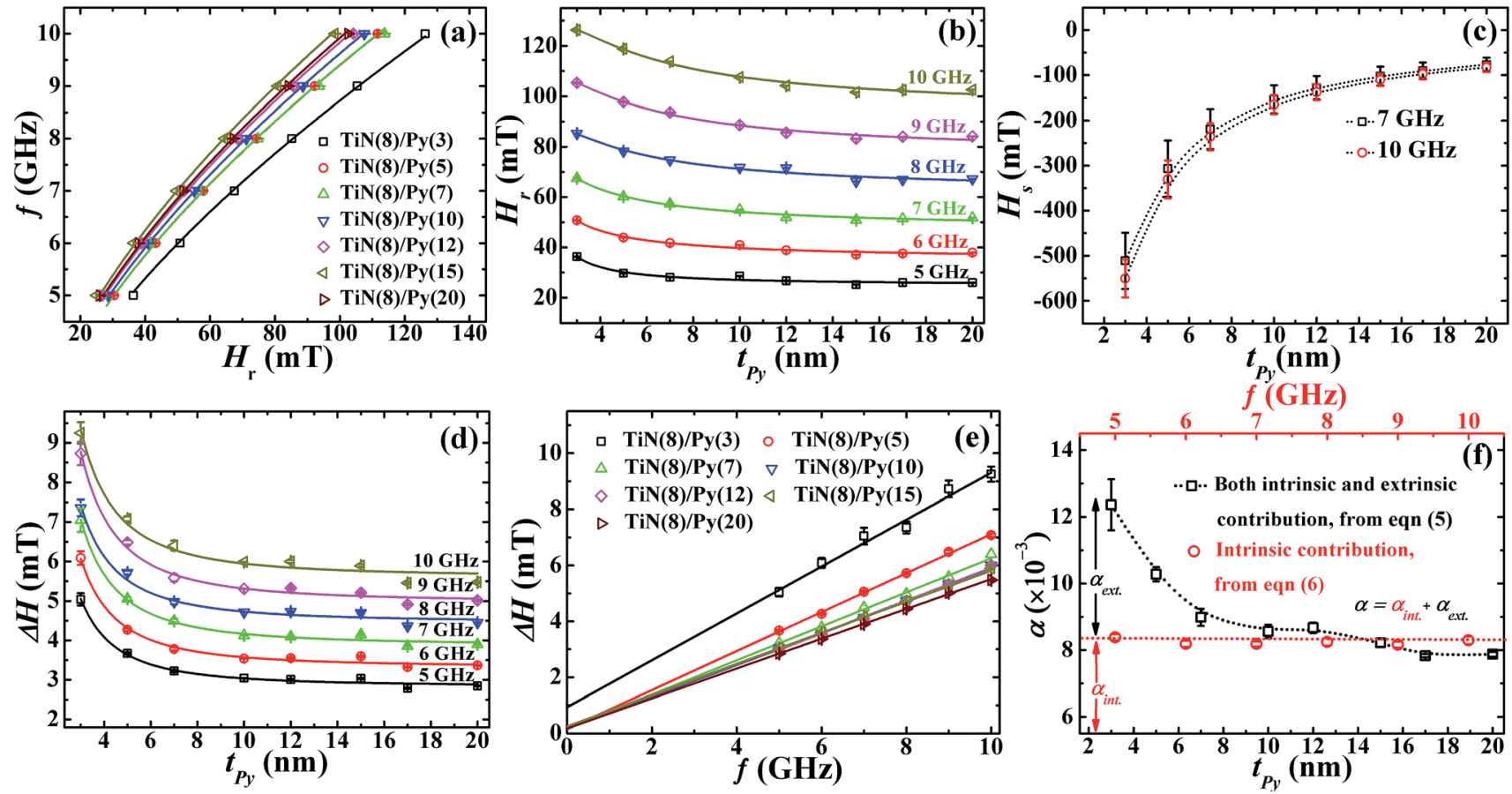

Fig. 3 The experimental data (open symbols) of (a) the frequency $(f)$ dependence in-plane resonance field $\left(H_{\mathrm{r}}\right)$ i.e. $f$ vs. $H_{\mathrm{r}}$ for TiN $(8 \mathrm{~nm}) / \mathrm{Py}\left(t_{\mathrm{Py}}\right)$ series of samples, and solid lines show the fitting according to Kittel's eqn (2). (b) Thickness dependence of resonance field $H_{r} i . e$. $H_{r} v s$. $t_{P y}$ at different constant resonance frequencies and solid lines show the fitting using eqn (4). (c) The thickness dependence of $H_{\mathrm{S}}$ i.e. $H_{\mathrm{S}} v s$. $t_{\mathrm{Py}}$ is obtained from the fitting using eqn (4) for TiN $(8 \mathrm{~nm}) / \mathrm{Py}\left(t_{\mathrm{Py}}\right)$ at different constant resonance frequencies. (d) Thickness dependence of $\Delta H$ i.e. $\Delta H$ vs. $t_{\mathrm{Py}}$ for TiN $(8 \mathrm{~nm}) / \mathrm{Py}\left(t_{\mathrm{Py}}\right)$ at different constant frequencies, and solid lines show the fitting using eqn (6). (e) The frequency dependence of line broadening $\Delta H$ i.e. $\Delta H$ vs. $f$ for different $t_{\text {Py }}$ samples, and solid lines are fitting using eqn (5). (f) The effect of $t_{\text {Py }}$ on the overall damping constant $\alpha$ (open black squares) obtained from eqn (5) and the intrinsic Gilbert damping constant $\alpha_{\text {int. }}$ (red open circles) obtained from eqn (6) for the epitaxial Py films. Note that the $\alpha_{\text {ext. }}$ damping comes from the extrinsic TMS contribution and can be inferred by using $\alpha=\alpha_{\text {int. }}+\alpha_{\text {ext. }}$ (the dotted lines are guide to the eye). 
where the $2^{\text {nd }}$ term in the eqn (3) represents the TMS induced shift in the resonance field, and is well explained by theory given by Arias and Mills. ${ }^{32}$ The " $r$ " is the magnon renormalization factor which has units of $\mathrm{T}^{-1}$. However, in our case $H_{\mathrm{r}} \ll$ $4 \pi M_{\mathrm{s}}$, therefore the eqn (3) can be rewritten as;

$$
H_{\mathrm{r}}=\frac{\left[\left(\frac{2 \pi f}{\gamma}\right)+r H_{\mathrm{S}}^{2}\right]^{2}}{\left(H_{\mathrm{S}}+4 \pi M_{\mathrm{S}}\right)}
$$

The $t_{\mathrm{Py}}$ dependence of $H_{\mathrm{r}}$ at different constant frequencies in 5-10 GHz range is fitted using this eqn (4), as shown by solid lines in Fig. 3(b). The various fitting parameters are presented in Table 1 of ESI file. $\dagger$ The fitted values of ' $r$ ' lie in the range of 8.85 $\times 10^{-5} \mathrm{mT}^{-1}$ to $2.10 \times 10^{-4} \mathrm{mT}^{-1}$. These values are close to the value $8 \times 10^{-5} \mathrm{mT}^{-1}$ reported previously on the basis of theory that accounts for the field shift data. ${ }^{32,40}$ The estimated values of $H_{\mathrm{S}}$ are found to change from $-551 \mathrm{mT}$ to $-82 \mathrm{mT}$ for $10 \mathrm{GHz}$ frequency (and from -511 to $-77 \mathrm{mT}$ for $7 \mathrm{GHz}$ frequency) as the $t_{\mathrm{Py}}$ is increased from 3 to $20 \mathrm{~nm}$, as shown in Fig. 3(c). While a gradual decrease in $H_{\mathrm{r}}$ and $H_{\mathrm{S}}$ are observed with increase in $t_{\mathrm{Py}}$ in low thickness regime (3-10 $\mathrm{nm}$ range), the changes in $H_{\mathrm{r}}$ and $H_{\mathrm{S}}$ become insignificant as $t_{\mathrm{Py}}$ is increased above $\sim 12 \mathrm{~nm}$. In the previous study, ${ }^{33}$ the higher $H_{\mathrm{S}}$ value observed at lower $t_{\mathrm{Py}}$ is attributed to the presence of local microscopic surface inhomogeneities in the sample. Thus, the observed dependencies of $H_{\mathrm{r}}$ and $H_{\mathrm{S}}$ below $10 \mathrm{~nm}$ and above $12 \mathrm{~nm} t_{\mathrm{Py}}$ thicknesses can be linked to the respective occurrence of non-uniform and uniform precession modes depending upon the film microstructure. In the non-uniform precession mode, the magnetic moments precess incoherently by the exchange between the resultant field due to dipolar interactions and in-plane surface anisotropy field, which leads to the generation of degenerate spin waves. These spin waves are responsible for the observed non-linear increase in $H_{\mathrm{r}}$ as $t_{\mathrm{Py}}$ is decreased below $12 \mathrm{~nm}$ (Fig. 3(b)). Understandably, the reduction in surface anisotropic field for $t_{\mathrm{Py}}>10 \mathrm{~nm}$ significantly enhances the uniform precession mode. In such a situation, the magnetic moments of the FM layer precess coherently, presumably as a result of relatively smaller influence of local microscopic surface inhomogeneities for $t_{\mathrm{Py}}>10 \mathrm{~nm}$ giving rise to thickness independent $H_{\mathrm{r}}$. It is to be pointed out here that the origin of the TMS, i.e. TMS noise observed in STT devices and the magnetic noise observed in Magnetic Tunnel Junctions (MTJs) ${ }^{\mathbf{4 1 - 4 3}}$ are fundamentally same as both are manifestations of the presence of local microscopic surface inhomogeneities, defects, magnetization fluctuations from domain wall hopping, etc. This noise contribution usually increases as the magnetic volume of the soft FM layer is decreased. ${ }^{41-43}$ Li et al., also found that the magnetization fluctuations can arise due to the formation of the top antiferromagnetic $\mathrm{NiO}^{43}(\sim 1 \mathrm{~nm})$ layer on epi-Py which is inevitably formed during the exposure to ambient environment while the sample is transferred for performing the subsequent measurement.

We now show that the other important experimental parameter, i.e., line-width $\Delta H\left(t_{\mathrm{Py}}\right)$ follows qualitatively similar trend as that of $H_{\mathrm{r}}\left(t_{\mathrm{Py}}\right)$ (see Fig. 3(d)). It should be noted that the dependence of $\Delta H$ on $t_{\mathrm{Py}}$ via $H_{\mathrm{S}}$ (since $H_{\mathrm{S}}=\frac{2 K_{\mathrm{S}}}{M_{\mathrm{S}} t_{\mathrm{Py}}}$ ) is also associated with the magnetization relaxation process, and therefore $\Delta H$ also depends upon the nature of resonance precession mode. Thus the increase in $\Delta H$ for $t_{\mathrm{Py}}<12 \mathrm{~nm}$ is also related to the finite contributions from the TMS. We shall now quantitatively separate the contributions to the overall damping constant " $\alpha$ " coming from intrinsic (i.e., the Gilbert's damping due to magnon-electron scattering) mechanism and the extrinsic TMS mechanism. These two contributions will be indicated by $\alpha_{\text {int. }}$ and $\alpha_{\text {ext. }}$, respectively. First, we fit the experimental $\Delta H(f)$ data for different $t_{\mathrm{Py}}$ (Fig. 3(e)) using the standard eqn $^{44}$

$$
\Delta H=\Delta H_{0}+4 \pi \alpha f / \gamma
$$

where $\Delta H_{0}$ is the zero frequency intercept which is understood to be an extrinsic inhomogeneous contribution to $\Delta H$. The $\Delta H(f)$ data is found to be well fitted for all $t_{\mathrm{Py}}$. The extrinsic linewidth $\Delta H_{0}$ is found to decrease from $0.93 \mathrm{mT}$ to $0.18 \mathrm{mT}$ with increase in $t_{\mathrm{Py}}$. In addition, it is found that as $t_{\mathrm{Py}}$ is increased from $3 \mathrm{~nm}$ to $20 \mathrm{~nm}$ the overall damping parameter $\alpha$ decreases (from $0.0124( \pm 0.0007)$ to $0.0079( \pm 0.0001))$ until $t_{\mathrm{Py}}$ increases to $10 \mathrm{~nm}$, and thereafter becomes more or less constant within the experimental errors (black open squares in Fig. 3(f)). In order to obtain the separate estimates of $\alpha_{\text {int. }}$ and $\alpha_{\text {ext. }}$, we revert to the theoretical model proposed by Arias and Mills ${ }^{32,40}$ according to which,

$$
\Delta H=\frac{32 S H_{\mathrm{S}}{ }^{2}}{\pi D} \sin ^{-1}\left(\frac{H_{\mathrm{r}}{ }^{1 / 2}}{\left(H_{\mathrm{r}}+4 \pi M_{\mathrm{s}}+H_{\mathrm{S}}\right)^{1 / 2}}\right)+\frac{4 \pi f}{\gamma} \alpha_{\text {int. }}
$$

where, $D$ is the exchange stiffness constant $\left(=2.0 \times 10^{-11} \mathrm{~T} \mathrm{~m}^{2}\right)$ and $S$ is a geometrical factor which is a characteristic of the surface roughness. While the first term in the eqn (6) accounts for the extrinsic factor which is responsible for the TMS, the second term is associated with intrinsic Gilbert damping constant $\alpha_{\text {int. }}{ }^{32,40,45,46}$ When the $\Delta H\left(t_{\mathrm{Py}}\right)$ data of Fig. $3(\mathrm{~d})$ is fitted using eqn (6), the fitted values of intrinsic damping constant $\alpha_{\text {int. }}$ so obtained for various films (with different $t_{\mathrm{Py}}$ ) are found to be nearly constant at $0.0083 \pm 0.0002$ (red circle symbols in Fig. 3(f)). However, it may be pointed out that intrinsic damping constant value, $\alpha_{\text {int. }} \sim 0.0083$, of the investigated films are still bit far away from the bulk like damping constant value, $\alpha_{\mathrm{B}} \sim$ 0.007 , of Py system. All the fitting parameters of eqn (6) are shown in Table 2 of ESI file. $\dagger$ We have also plotted the $1 / t_{\mathrm{Py}}{ }^{2}$ dependence of $\Delta H$ i.e. $\Delta H v$ s. $1 / t_{\mathrm{Py}}{ }^{2}$ and solid lines are fitted with eqn (6). The $\Delta H$ vs. $1 / t_{\mathrm{Py}}{ }^{2}$ dependency is compared with $\Delta H v s$. $t_{\mathrm{Py}}$ dependency (see Fig. 2 and 3 of ESI file $\dagger$ ). In all the cases, the $S$ values are almost same with previous values within the error of measurement as determined by using eqn (6), (see Table 2 of ESI file $\dagger$ ). This $t_{\mathrm{Py}}$ independence of the ' $S$ ' parameter values indicate that the scattering centers are not changing with the change in the thickness of Py layer which is also consistence with Arias and Mills theoretical model. The $t_{\mathrm{Py}}$ dependence of ' $S$ ' parameter values are nearly constant therefore, in thickness 
regime (with non-uniform precession) especially at lower $t_{\mathrm{Py}}<$ $12 \mathrm{~nm}$ in our case, the increase in TMS with decrease in $t_{\mathrm{Py}}(<12$ $\mathrm{nm}$ ) is due to the increase in $H_{\mathrm{S}}$ values. From Fig. 3(f), it can be seen that the intrinsic damping regime is almost clearly separated from the low thickness regime where the extrinsic damping resulting from TMS contributions starts appearing as $t_{\mathrm{Py}}$ is reduced below $12 \mathrm{~nm} .^{32,40,45,46}$

From the foregoing discussion, it is concluded that TMS is negligible in 2-D Py samples of thickness greater than $10 \mathrm{~nm}$, and the Arias and Mills theoretical model satisfactorily accounts for the negligible presence of TMS. Thus, a $12 \mathrm{~nm}$ thick film is sufficiently thick to reduce the influence of TMS to an extent that is sufficiently small to be negligible at least within the experimental resolution. We have therefore chosen the $12 \mathrm{~nm}$ epitaxial Py sample to further explore the effect of the thickness of $\beta$-Ta layer (placed over the epitaxial Py layer) on effective Gilbert's damping constant, which is discussed in the remaining part of the paper. For this study we have grown the $\beta$ Ta layer of different thicknesses over the $12 \mathrm{~nm}$ epitaxial Py layer, and the detailed results are as follows.

\section{Thickness dependent $\alpha_{\text {eff }}$ in $\operatorname{TiN}(200) / P y(200) / \beta-T a$ multilayer thin films}

In this study, the effect of change in thickness of $\beta$-Ta film (1.5$15 \mathrm{~nm}$ ) deposited over the optimized Py layer (2-D epi-Py of 12 $\mathrm{nm}$ ) on spin pumping mechanism is explored. Fig. 4 shows the $\mathrm{X}$-ray diffraction patterns recorded in $\theta-2 \theta$ mode for $\beta$-Ta (62 $\mathrm{nm}), \mathrm{Si} / \mathrm{TiN}(8 \mathrm{~nm}) / \mathrm{Py}(12 \mathrm{~nm}) / \beta$-Ta $(7.5 \mathrm{~nm}), \mathrm{Si} / \mathrm{TiN}(8 \mathrm{~nm}) / \mathrm{Py}$ $(12 \mathrm{~nm}) / \beta$-Ta $(10.5 \mathrm{~nm}), \mathrm{Si} / \mathrm{TiN}(8 \mathrm{~nm}) / \mathrm{Py}(12 \mathrm{~nm}) / \beta$-Ta $(15$ $\mathrm{nm}$ ) and compared with the JCPDS (joint committee on powder

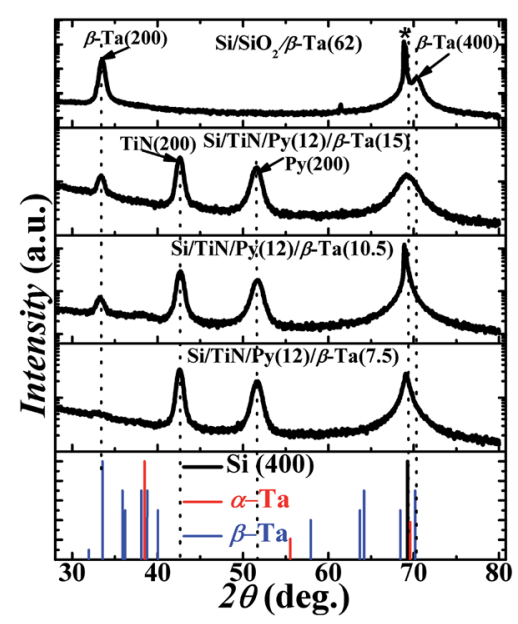

Fig. 4 Bottom panel shows the standard XRD pattern as per JCPDS file for Ta thin films. The blue lines are the peak locations for $\beta$-Ta phase and red lines are for $\alpha$-Ta phase. Black line corresponds to $\mathrm{Si}(400)$. The top panel shows the $\theta-2 \theta \mathrm{X}$-ray diffraction (XRD) pattern of pure $\mathrm{Ta}$ thin film grown on $\mathrm{Si}(100)$ substrate having native $\mathrm{SiO}_{2}$ layer. In between the top and bottom panels, the other three panels are $\theta-2 \theta$ XRD patterns for Si/TiN/Py/ $\beta$-Ta $\left(t_{\mathrm{Ta}}=7.5,10.5,15 \mathrm{~nm}\right)$ bilayers with Py thickness of $12 \mathrm{~nm}$. The peaks from (200) planes belonging to Py and TiN are also indicated. The star symbol highlights the (400) peak from Si substrate. diffraction standards) files (pdf\#191290 \& pdf\#040788) of Ta shown in bottom panel. The growth of Ta has been done at high power $(150 \mathrm{~W})$ to ensure formation of the desired tetragonal $\beta$ Ta phase. The observed $2 \theta$ peak position at $33.4^{\circ}$ corresponds to $d$ value of $2.67 \AA$, that matches with the reported values. ${ }^{47}$ It reveals that the Ta thin films have grown in desired tetragonal $\beta$ phase oriented preferentially along (200), instead of the body centered cubic $\alpha$-Ta phase. The (400) peak of $\beta$-Ta in all Si/TiN (8 $\mathrm{nm}) / \mathrm{Py}(15 \mathrm{~nm}) / \beta$-Ta $\left(t_{\mathrm{Ta}} \mathrm{nm}\right)$ samples is not clearly distinguishable as it merged with $\mathrm{Si}(400)$ due to low thickness of Ta.

The FMR spectra of TiN $(8 \mathrm{~nm}) / \mathrm{Py}(12 \mathrm{~nm}) / \beta$-Ta $\left(t_{\mathrm{Ta}} \mathrm{nm}\right)$ samples are recorded at different constant frequencies in the range 5-10 GHz. For clarity and conciseness, typical representative FMR spectra recorded at $9 \mathrm{GHz}$ frequency for different $t_{\mathrm{Ta}}$ samples are shown in Fig. 5(a). The various FMR spectra are fitted with derivative of Lorentzian function as shown by solid lines in Fig. 5(a), and the values of fitting parameters $H_{\mathrm{r}}$ and $\Delta H$ are determined at different constant frequencies. The frequency dependence of observed $H_{\mathrm{r}}$ for various TiN $(8 \mathrm{~nm}) / \mathrm{Py}(12 \mathrm{~nm}) / \beta$ Ta $\left(t_{\mathrm{Ta}} \mathrm{nm}\right)$ samples is shown in Fig. $5(\mathrm{~b})$. The values of $H_{\mathrm{r}}$ are found to be nearly constant irrespective of $\beta$-Ta layer thicknesses (i.e. independent of $t_{\mathrm{Ta}}$ ), at all the frequencies as shown in Fig. 5(c). This suggests the negligible presence of extrinsic TMS contribution to the overall damping in these layers. The $f$ vs. $H_{\mathrm{r}}$ data are fitted using the Kittel's eqn (2); $f=\frac{\gamma}{2 \pi}\left[\left(H_{\mathrm{r}}\right)\left(H_{\mathrm{r}}+4 \pi M_{\text {eff }}\right)\right]^{\frac{1}{2}}$, and fits are shown by solid lines in Fig. 5(b). The estimated values of $4 \pi M_{\text {eff }}$ are nearly constant within experimental error for all different $t_{\mathrm{Ta}}$ layers (see Fig. ESI5(b) $\dagger$ ). However these constant values are slightly higher than $4 \pi M_{\text {eff }}$ value of Py (12 $\mathrm{nm}$ ) layer. This slight increase in $4 \pi M_{\text {eff }}$ might be result of either the magnetic proximity effect, ${ }^{\mathbf{2 6 , 4 8 , 4 9}}$ or the interface modification during the $\beta$-Ta layer deposition over Py layer. To explore it further we have plotted the $4 \pi M_{\text {eff }} v s .1 / t_{\mathrm{Py}}$ data for $\operatorname{Si} / \mathrm{TiN}(8 \mathrm{~nm}) / \mathrm{Py}\left(t_{\mathrm{Py}}=3-20 \mathrm{~nm}\right)$ and $\mathrm{Si} / \mathrm{TiN}(8 \mathrm{~nm}) / \mathrm{Py}\left(t_{\mathrm{Py}}=3-20 \mathrm{~nm}\right) / \beta-\mathrm{Ta}(6)$, respectively in Fig. 5(d). We have observed that there is higher slope when there in the absence of Ta layer. Even the $y$-axis intercept $4 \pi M_{\mathrm{s}}$ is higher for $\mathrm{Si} / \mathrm{TiN}(8 \mathrm{~nm}) / \mathrm{Py}\left(t_{\mathrm{Py}}=3-20 \mathrm{~nm}\right) / \beta-\mathrm{Ta}(6)$ as compared to $\mathrm{Si} / \mathrm{TiN}(8 \mathrm{~nm}) / \mathrm{Py}\left(t_{\mathrm{Py}}=3-20 \mathrm{~nm}\right)$. It clearly confirms that the higher value of $4 \pi M_{\mathrm{s}}$ might be due to the additional magnetization added into it due to addition of $\beta$-Ta layer, though $\beta$-Ta induced changes in surface anisotropy is also observed which is however expected. However these changes are independent of $t_{\mathrm{Ta}}$ layers as evident from the constant values of $4 \pi M_{\text {eff }}$ for all different $\beta$-Ta layers. We have also studied the possibility of magnetic dead layer formation which can also affect the saturation magnetization in epi-Py/ $\beta$-Ta interface due to the presence of $\beta$-Ta layer adjacent to epi-Py layer as seen by other groups. ${ }^{11}$ However, such issue is not seen in the present samples. If dead layer is supposed to be formed in epi-Py/ $\beta$-Ta interface, then there would must be a decrease in magnetization in-terms of emu $\mathrm{cm}^{-3}$ due to over estimation of volume of the epi-Py in epi-Py/ $\beta$-Ta, which is not supported by the effective magnetization of samples inferred using FMR. To further strengthen the absence of dead layer in our studied samples we have performed the $M v s$. $H$ measurement on epi-Py $(3-20 \mathrm{~nm}) /$ 

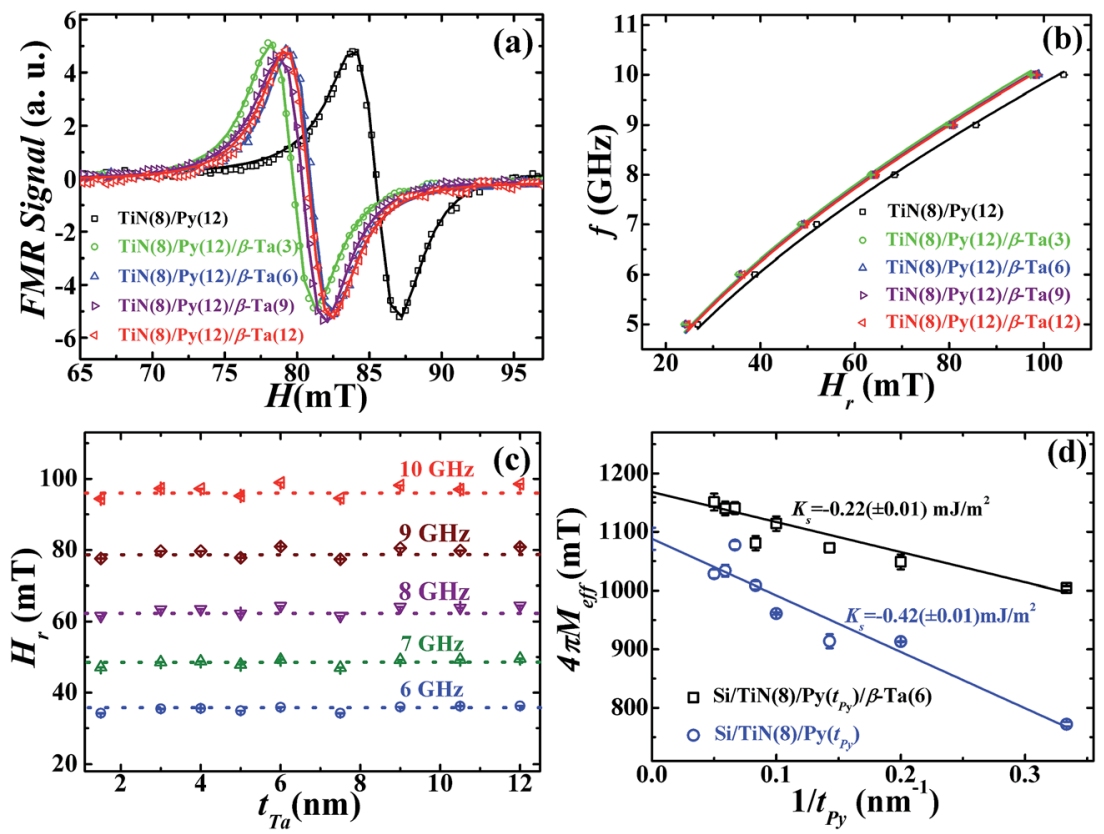

Fig. 5 (a) The derivative FMR spectra (recorded at $9 \mathrm{GHz}$ ) of the different samples of TiN $(8 \mathrm{~nm}) / \mathrm{Py}(12 \mathrm{~nm}) / \mathrm{Ta}\left(t_{\mathrm{Ta}}\right)$ series, where the Ta layer thickness $t_{T a}$ varies from 1.5 to $15 \mathrm{~nm}$. Symbols represent experimental points and solid lines show the fit using eqn (1). (b) The frequency (f) vs. inplane resonance field $\left(H_{r}\right)$ dependence for samples with different $t_{T a}$ and solid lines shows the fit employing eqn (2). (c) The $t_{T a}$ dependence of $H_{r}$ i.e. $H_{\mathrm{r}} v$ s. $t_{\mathrm{Ta}}$ for TiN $(8 \mathrm{~nm}) / \mathrm{Py}(12 \mathrm{~nm}) / \mathrm{Ta}\left(t_{\mathrm{Ta}}\right)$ samples corresponding to different constant resonance frequencies. The $f v s$. $H_{\mathrm{r}}$ plots for samples with $t_{\mathrm{Ta}}=1.5,4,5,7.5$, and $10.5 \mathrm{~nm}$, refer Fig. $4 \mathrm{a}$ of the ESI $\dagger$ file. $\dagger$ (d) $4 \pi M_{\text {eff }} v s .1 / t_{\text {Py }}$ plots for Si $/ T i N(8 \mathrm{~nm}) / \mathrm{Py}\left(t_{\mathrm{Py}}=3-20 \mathrm{~nm}\right)$ and Si/TiN $(8 \mathrm{~nm}) / \mathrm{Py}$ $\left(t_{\mathrm{Py}}=3-20 \mathrm{~nm}\right) / \beta-\mathrm{Ta}(6)$ multilayer thin films. Open symbols are experimental data and solid lines are fit to experimental data by using expression $4 \pi M_{\mathrm{eff}}=4 \pi M_{\mathrm{S}}+\frac{2 K_{\mathrm{S}}}{M_{\mathrm{s}} t_{\mathrm{Py}}}$.

$\beta$-Ta $(6 \mathrm{~nm})$ series of samples. We have plotted the $\left(M_{\mathrm{s}} \times t_{\mathrm{Py}}\right) v s$. $t_{\mathrm{Py}}(3-20 \mathrm{~nm})$ and congruently linearly fitted the data as shown Fig. 6, the zero intercept of $M_{\mathrm{s}} \times t_{\mathrm{Py}} v s$. $t_{\mathrm{Py}}(3-20 \mathrm{~nm})$ data evidently affirms the absence of dead-layer formation in these samples.

Fig. 7(a) shows the observed frequency dependence of $\Delta H$ (open data symbols) and the fitted curves (solid lines) using eqn (5), which shows that $\Delta H$ increases linearly with the resonance frequency. The inhomogeneous broadening, $\Delta H_{0} \sim 0.27-0.37$ $\mathrm{mT}$, in $\operatorname{Py}(12) / \beta-\mathrm{Ta}(6,9,12 \mathrm{~nm})$ samples are bit higher as compare to the bare $\operatorname{Py}(12)$, and this behavior is matching excellently well with those values reported in literatures. ${ }^{26}$ The difference observed in the $\Delta H_{0}$ in samples with and without

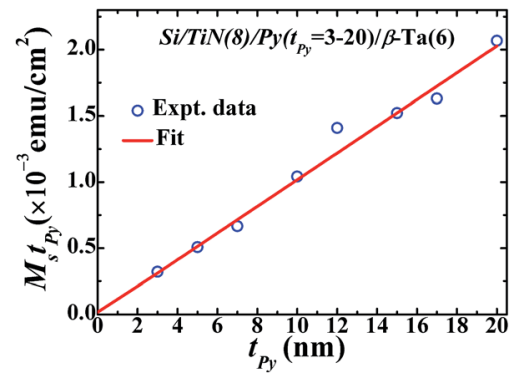

Fig. 6 Thickness dependent saturation magnetization per unit area $\left(M_{s} t_{\text {Py }} v s . t_{\text {Py }}\right)$ plot of epitaxial Si-TiN $(8 \mathrm{~nm}) / \mathrm{Py}\left(t_{\mathrm{Py}}\right) / \beta-\mathrm{Ta}(6 \mathrm{~nm})$. Open symbols are experimental data and solid line is $t$ linear fit.
$\beta$-Ta over layer can be attributed to the finite surface modification of the underlying Py layer as more and more heavy Ta atoms are sputtered over Py. Consistent with the results of Fig. $5(\mathrm{c})$, the linear increase of $\Delta H$ with $f$ clearly suggests that the damping of the precession in this $\beta$-Ta capped bilayer system is largely governed by the intrinsic Gilbert's phenomena. Therefore, the contributions in the damping from an extrinsic TMS effects are expected to be very small. The variation of the estimated values of effective Gilbert damping constant $\alpha_{\text {eff }}(=\alpha+$ $\left.\Delta \alpha\left(t_{\mathrm{Ta}}\right)\right)$ with $\beta$-Ta layer thickness is shown in Fig. 7(b). Here $\alpha$ is the damping constant for $\mathrm{Si} / \mathrm{TiN}(8 \mathrm{~nm}) / \mathrm{Py}(12 \mathrm{~nm})$ and $\Delta \alpha\left(t_{\mathrm{Ta}}\right)$ term comes due to the spin pumping from the adjacent Ta layer in the TiN $(8 \mathrm{~nm}) / \mathrm{Py}(12 \mathrm{~nm}) / \beta$-Ta $\left(t_{\mathrm{Ta}} \mathrm{nm}\right)$ bilayers. It is evident that the observed values of $\alpha_{\text {eff }}\left(t_{\mathrm{Ta}}\right)$ for these bilayers are smaller than the damping constant found for the bare TiN (8 nm)/Py (12 nm) sample (Fig. 7(b)). This is quite anomalous because the spin pumping in a FM/NM system is usually accompanied with either significant increase in $\alpha_{\text {eff }}$ (e.g., when $\mathrm{NM}=\mathrm{Pt}, \mathrm{Pd}$, etc. $)^{\mathbf{2 , 3 , 6 , 2 1 , 2 3}}$ or negligible increase in $\alpha_{\text {eff }}(e . g$., when $\mathrm{NM}=\mathrm{Ta}, \mathrm{Au}$, $\mathrm{Cu}, \mathrm{Al}$, etc.). ${ }^{\mathbf{2 , 3 , 5 , 6 , 2 1 , 2 3}}$ In addition to the observed anomalous decrease of $\alpha_{\text {eff }}\left(t_{\mathrm{Ta}}\right)$ it also exhibits a minimum near $t_{\mathrm{Ta}} \approx 6 \mathrm{~nm}$ $\left(\beta\right.$-Ta $\left.(4 \mathrm{~nm})+\mathrm{Ta}_{2} \mathrm{O}_{5}(2 \mathrm{~nm})\right)($ Fig. $7(\mathrm{~b}))$ which is discussed in forthcoming section.

We now turn to the different aspects of spin pumping from materials perspective, particularly the choice of NM metal. In the present case of TiN $(8 \mathrm{~nm}) / \mathrm{Py}(12 \mathrm{~nm}) / \beta-\mathrm{Ta}\left(t_{\mathrm{Ta}} \mathrm{nm}\right)$ bilayers, owing to the smaller spin-flip rate compared to the rate of spin- 

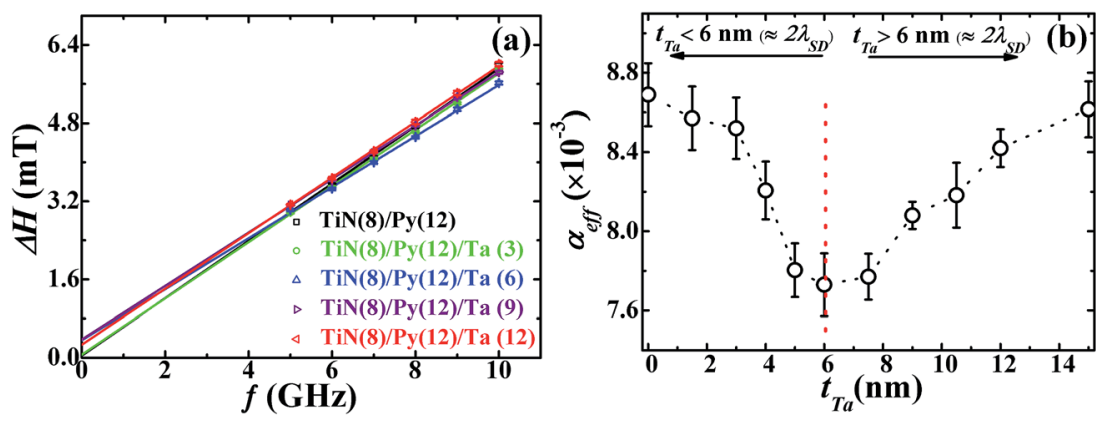

Fig. 7 (a) Variation of $\Delta H$ with $f$ i.e. $\Delta H$ vs. $f$ at different constant $t_{\mathrm{Ta}}$ and the solid lines fits using eqn (5). Here the Ta layer thickness $t_{\mathrm{Ta}}$ varies from 1.5 to $15 \mathrm{~nm}$. (b) The effective Gilbert damping constant $\left(\alpha_{\text {eff }}\right)$ obtained from the fitting using eqn (5). The dotted lines are guide to the eyes. The $\Delta H$ vs. $f$ plots for samples with $t_{T a}=1.5,4,5,7.5$, and $10.5 \mathrm{~nm}$, refer Fig. $4(\mathrm{~b})$ of the $\mathrm{ESI} \uparrow$ file. $\dagger$

injection from the Py layer, the $\beta$-Ta layer can be assumed to act as non-magnetic reservoir. ${ }^{22}$ Under this condition, when the magnetization $\vec{m}$ precesses around the effective applied magnetic field, a transfer of spin angular momentum $(\vec{S})$ from Py to the Ta layer is expected. This transfer of $\vec{S}$ only takes place if the thickness of the FM layer is greater than its transverse spin coherence length, which is typically $<1 \mathrm{~nm}$ in case of transition metal elements such as $\mathrm{Co}, \mathrm{Ni}, \mathrm{Fe}$, etc. ${ }^{22,25}$ The magnetization precession in the FM layer induces a torque, which governs the current density of spin injection $j_{\mathrm{S}}$ from Py to $\beta$-Ta layer. ${ }^{21-25,50,51}$ The spin current density $\overrightarrow{j_{\mathrm{s}}}$ can be written as:

$$
\overrightarrow{j_{\mathrm{S}}}=\frac{\hbar}{2} \operatorname{Re}\left(g^{\uparrow \downarrow}\right) \vec{m} \times \frac{\mathrm{d} \vec{m}}{\mathrm{~d} t}
$$

where $\vec{m}$ is the unit vector along Py layer's magnetization, and $\operatorname{Re}\left(g^{\uparrow \downarrow}\right)$ is the real-part of the spin mixing conductance $g^{\uparrow \downarrow}$ which governs the transportation efficiency of spin current through the interface. In the present case, the imaginary part of $g^{\uparrow \downarrow}$ is expected to be insignificant $t^{51}$ because of the observed very weak dependence of the resonance field on the $\beta$-Ta layer thickness (Fig. 5(c)) and hence can be neglected. The spin current generated by magnetization-precession (i.e., spin pumping) is polarized perpendicular both to the instantaneous magnetization $\vec{m}$ and its time derivative $\frac{\mathrm{d} \vec{m}}{\mathrm{~d} t}$. It is to be recalled here that this transfer of $\vec{S}$ from FM to NM layer is known to depend critically upon the nature of the NM layer via a probability parameter $\varepsilon$ which is related to the change of the spin orientation of the conduction electrons due to the momentum transfer, and is governed by the ratio of spin elastic scattering time to spin flip time, i.e. $\varepsilon=\frac{\tau_{\mathrm{el}}}{\tau_{\mathrm{SF}}}$. The $\varepsilon$ can also be expressed in terms of fine structure constant $\alpha_{\text {fine }}$ and atomic number $Z_{\mathrm{NM}}$ of NM layer, i.e. $\varepsilon=\left(Z_{\mathrm{NM}} \alpha_{\text {fine }}\right)^{4}$. It may be noted that the $\varepsilon$ value, $0.0805 \pm 0.0002$, for $\beta$-Ta ${ }^{52-54}$ is smaller compared to $\varepsilon$ value, $\geq 0.1049$, reported for Pt. ${ }^{53}$ Tserkovnyak et al. ${ }^{21,22,24,25}$ showed that in the case of $\varepsilon>0.1$, the spin accumulation at the interface is not possible in sharp contrast to the case when $\varepsilon<0.1$.

In the case for NM layer having $\varepsilon<0.1$, (e.g., $\beta$-Ta, as in the present case) and $t_{\mathrm{NM}} \leq \lambda_{\mathrm{SD}}$, the spin angular momentum $(\vec{S})$ associated with the spins accumulated near the Py $(12 \mathrm{~nm}) / \beta$-Ta interface creates a non-equilibrium spin density in $\beta$-Ta layer. ${ }^{21,23}$ However, according to spin pumping model of Tserkovnyak et al.,${ }^{21}$ the spin accumulation is expected to be insignificant for non-magnetic metal layer thickness higher than $\lambda_{\mathrm{SD}}$ due to the diffusive nature of the spin transport. We would also like to mention here that a substantial mismatch of Fermi surfaces of the FM and NM layers at the interface, which is also the case in the presently studied Py/ $\beta$-Ta bilayers, can also result in a non-equilibrium spin accumulation, as argued theoretically by Stiles et $a l .{ }^{30}$ As a consequence of a non-equilibrium spin density in $\beta$-Ta layer, a back flow of spin current (indicated by $\left.\overrightarrow{J_{S}^{0}}\right)$ into the Py layer takes place. This back flow opposes the spin current entering in to the $\beta$-Ta layer. Therefore, in such a case the net spin current $\overrightarrow{j_{\mathrm{S}}}(z)$ can be expressed as $\overrightarrow{j_{\mathrm{s}}}(z)=\overrightarrow{j_{\mathrm{S}}}+\overrightarrow{j_{\mathrm{S}}^{0}}$. The back flow spin current is expressed as $\overrightarrow{j_{\mathrm{S}}^{0}}=-\frac{g^{\uparrow \downarrow}}{4 \pi}\left[\vec{m} \times \overrightarrow{\mu_{\mathrm{N}}} \times \vec{m}\right]=\left[\overrightarrow{\mu_{\mathrm{N}}}-\vec{m}\left(\vec{m} \cdot \overrightarrow{\mu_{\mathrm{N}}}\right)\right] \cdot{ }^{22}$ Theoretically, it was proposed that accumulation of spins at the interface of such FM/NM systems is accompanied by absorption of spin angular momentum at the interface. ${ }^{30}$ It was further established that during this back flow, while the component of $\overrightarrow{J_{S}^{0}}$ parallel to the instantaneous magnetization $\vec{m}(t)$ of the FM layer effectively suppresses the spin pumping from FM layer, ${ }^{21-25,50,51}$ the interaction of the transverse component of $\overrightarrow{J_{\mathrm{S}}^{0}}$ with the in-plane magnetization of FM layer generates a torque ${ }^{23}$ which is however not sufficient to reduce the effective Gilbert damping constant $\left(\alpha_{\text {eff }}\right)$ under total angular momentum conservation. However the physical origin of this observed damping behavior can be understood as a result of net non-equilibrium spin accumulation linked with anti-damping toque on FM layer under total energy conservation. Before looking into this nonequilibrium spin accumulation linked with anti-damping we would like to remark that during FMR, the spin current injected by FM layer into the NM is converted into the charge current by ISHE. The efficiency of this conversion depends on the spin flip probability of the NM material. In case of $\beta$-Ta this spin to charge conversation efficiency is low due to its low value of spin flip probability $(0.08)^{52-54}$ as compared to higher value for Pt $(0.10) .{ }^{55}$ As a result $\beta$-Ta accumulates more spins up to $\lambda_{\mathrm{SD}}$ compared to Pt which is a spin sink material. However, the 
small percentage change of spin current into charge current in $\beta$-Ta is responsible for a weak source for in-plane charge current. The strength of this in-plane charge current density starts gradually decreasing when $t_{\mathrm{Ta}}$ exceeds $\lambda_{\mathrm{SD}}$. This significant amount of in-plane charge current is responsible for Rashba spin-orbit torque at interface; due to the structural inversion asymmetry of the two dissimilar materials at the interface of $\mathrm{Py} / \beta$-Ta, in the presence this in-plane charge current due to ISHE, the spin-orbit Hamiltonian breaks the degeneracy of the electron spin states near the interface, creating a nonequilibrium spin-accumulation, known as Rashba spin-orbit torque.

Therefore a net non-equilibrium spin accumulation results from, (i) Rashba spin-orbit torque at interface (ISOC contribution), (ii) spin pumping induced spin accumulation in $\beta$-Ta, up to $\lambda_{\mathrm{SD}}$ (BSOC contribution), and (iii) interfacial Fermi surface mismatch. This net non-equilibrium spin accumulation exerts a torque on local magnetization of FM layer. The spin torque is given by ${ }^{11,12} \vec{T}=\frac{\vec{S} \times \hat{m}}{\tau_{\Delta}}+\frac{\hat{m} \times \vec{S} \times \hat{m}}{T_{\mathrm{xc}}}$, or more generally the net spin torque is given by, $\vec{T}=T_{\perp} \hat{S} \times \hat{m}+T_{\|} \hat{m} \times \hat{S} \times \hat{m}$, where $\vec{S}$ is net non-equilibrium spin density, $T_{\perp}$ is out of plane torque or field-like torque, $T_{\|}$is in-plane torque or anti-damping torque, $\tau_{\Delta}$ characterize the time scale of the precession of the spin density around the magnetization, and $T_{\mathrm{xc}}$ is the parameter associated with the momentum relaxation rate $\left(\frac{1}{\tau}\right)$ due to spinindependent scattering, ferromagnetic exchange splitting $\left(\Delta_{\mathrm{xc}}\right)$ and spin relaxation time $\left(\tau_{\mathrm{sf}}\right)$. The in-plane torque arises from a change of the spin density induced by its precession around the exchange field. However, in presence of spin accumulation $\left(\tau_{\mathrm{sf}} \rightarrow \infty\right)$, anti-damping to field-like torque ratio can be represented as, $\frac{T_{\|}}{T_{\perp}} \approx \tau \frac{\Delta_{\mathrm{xc}}}{4}$. Here field-like torque strength is negligible since the values of $H_{\mathrm{r}}$ are independent of $t_{\mathrm{Ta}}$. Nevertheless the field-like torques usually become relatively strong at lower thickness $(<7 \mathrm{~nm})$ of FM layer. ${ }^{56}$ As the $t_{\mathrm{NM}}$ increases upto $t_{\mathrm{Ta}}=6 \mathrm{~nm} \approx\left(\sim 2 \lambda_{\mathrm{SD}}\right.$ of $\left.\mathrm{Ta}(\sim 4 \mathrm{~nm})+\mathrm{Ta}_{2} \mathrm{O}_{5}(\sim 2 \mathrm{~nm})\right)$ the net spins accumulation, interfacial and inside $\beta$-Ta, increases which results in escalation of the net spin accumulation induced inplane torque (anti-damping torque) on FM. Consequently, damping reduces till the $2 \lambda_{\mathrm{SD}}\left(+\mathrm{Ta}_{2} \mathrm{O}_{5}(\sim 2 \mathrm{~nm})\right)$ of $\beta$-Ta. However at $t_{\mathrm{Ta}} \geq 6 \mathrm{~nm} \approx\left(\sim 2 \lambda_{\mathrm{SD}}\right.$ of $\left.\mathrm{Ta}(\sim 4 \mathrm{~nm})+\mathrm{Ta}_{2} \mathrm{O}_{5}(\sim 2 \mathrm{~nm})\right)$ the net non-equilibrium spin accumulation starts diminishing because (i) spin coherence within the bulk of Ta dies out, ${ }^{53}$ and (ii) ISHE signal start diminishing which results an decrease in the Rashba spin-orbit torque at interface. As a result as $t_{\mathrm{NM}}$ increases beyond this thickness $(\sim 6 \mathrm{~nm})$ the net spin accumulation induced inplane torque on FM starts diminishing and leads to the increase in the Gilbert damping as shown in Fig. 7(b). Our results suggest that in $\mathrm{Py} / \beta$-Ta bilayer system the anti-damping torque dominates, i.e., $T_{\|} \gg T_{\perp}$. Therefore the precession of the spin density is along the exchange field, and the spin momentum relaxation rate is quite low which is however expected in the case of $\beta$-Ta due to its low conductivity or the presence of net nonequilibrium spin accumulation.
The observed thickness dependent $\alpha_{\text {eff }}$ in the TiN $(8 \mathrm{~nm}) / \mathrm{Py}$ $(12 \mathrm{~nm}) / \beta$-Ta $\left(t_{\mathrm{Ta}}\right)$ bilayers (Fig. 7(b)) can therefore be attributed to net non-equilibrium spin accumulation at the FM/NM interface and in the NM layer. It may however be noted here that Rashba like anti-damping torque exists predominantly in presence of uniform precession of magnetization. ${ }^{13,28-30}$ As established in the previous section, this is indeed the case with the TiN (8 nm)/Py (12 nm) stack. Therefore, the RSOT arising due to spin absorption occurring at the Py/ $\beta$-Ta interface along with the BSOC contributions are responsible for reduction in effective Gilbert damping constant $\alpha_{\text {eff }}$ from 0.0087 (in $t_{\mathrm{Ta}}=0$ ) to 0.0077 (in $t_{\mathrm{Ta}}=6 \mathrm{~nm}$ ), i.e., a change $\Delta \alpha\left(t_{\mathrm{Ta}}\right)=\alpha_{\text {eff }}-\alpha=$ -0.0010 occurs when $t_{\mathrm{Ta}}$ increases to $6 \mathrm{~nm}\left(\approx 2 \lambda_{\mathrm{SD}}\right)$. However we can't ignore the fact that reduction in the $\alpha_{\text {eff }}$ with an increase in $t_{\mathrm{NM}}$ might also be caused by a change of the gyromagnetic ratio, as expected in presence of spin pumping and a backflow, but to confirm it further detailed investigations in term of high frequency in-plane and out-plane FMR measurement are needed. ${ }^{23}$

In order to ascertain what constitutes the interface between top Ta layer and the underlying Py layer, we have carried out the X-ray photoelectron spectroscopy (XPS) studies of Ta/Py bilayers of various Ta thicknesses in the range belonging to the antidamping regime, i.e., $t_{\mathrm{Ta}}<6 \mathrm{~nm}$ regime. Fig. 8 shows the XPS spectra of Ta-4f for two bilayer samples having different Ta layer thicknesses, the lowest $1.5 \mathrm{~nm}$ used in our study (that is also about the minimal self-passivating thickness) and a sufficiently larger thickness of $t_{\text {Ta }}=4 \mathrm{~nm}$ (but less than $6 \mathrm{~nm}$ ). The XPS spectra of the epi-Py $(12 \mathrm{~nm}) / \beta$-Ta $(1.5 \mathrm{~nm})$ bilayer shown in Fig. $8(\mathrm{a})$, on deconvolution reveals Ta- $4 \mathrm{f}_{5 / 2}$ and Ta- $4 \mathrm{f}_{7 / 2}$ binding energy peaks for the oxidized tantalum at $28.16 \pm 0.03 \mathrm{eV}, 26.30$ $\pm 0.02 \mathrm{eV}$, metallic tantalum at $23.20 \pm 0.12 \mathrm{eV}$, and $21.32 \pm$ $0.05 \mathrm{eV}$, respectively. The position of peaks matches with the known peak positions. ${ }^{57}$ Fig. 8(b) compares the XPS spectra of bilayers with $t_{\mathrm{Ta}}=1.5$ and $4.0 \mathrm{~nm}$. We observe that the oxidized Ta-4f peaks almost remain the same, whereas the metallic Ta-4f peaks are much reduced in intensity in case of bilayer $t_{\mathrm{Ta}}=$ $1.5 \mathrm{~nm}$, the peak positions remaining same. It may be stressed here that lower peak intensity of the XPS signal due to metallic Ta compared to that due to Ta as oxide in a given specimen should not be directly taken as indication of their absolute thicknesses since in the XPS, which is highly surface sensitive technique, the signal from the surface oxide layer is expected to be far stronger than the protected Ta metal below the top oxide layer. Nevertheless, the relative comparison of the XPS data of Fig. 8 suggests that the passivating layer thickness of $\mathrm{Ta}_{2} \mathrm{O}_{5}$ remains just about the same with increasing Ta layer thickness, resulting in an increase in the balance Ta metal layer thickness. If the minimal Ta thickness of $\sim 1.5 \mathrm{~nm}$ oxidizes completely as suggested by the XPS study (and also from our previous XRR studies ${ }^{26}$ ) forming $\mathrm{Ta}_{2} \mathrm{O}_{5}$ then the Py layer makes direct contact with $\mathrm{Ta}_{2} \mathrm{O}_{5}$ (and not with Ta metal). Since $\mathrm{Ta}_{2} \mathrm{O}_{5}$ breaks the inversion symmetry, then due to Rashba effect it will show decrease in damping. The observed decrease in $\alpha$ is 0.0001 for $1.5 \mathrm{~nm}$ Ta layer (over the bare Py layer). However on increasing the Ta layer thickness to $4 \mathrm{~nm}$ (in which case Py layer is in contact with Ta metal), there is further decrease in $\alpha$ by 0.0004 . 

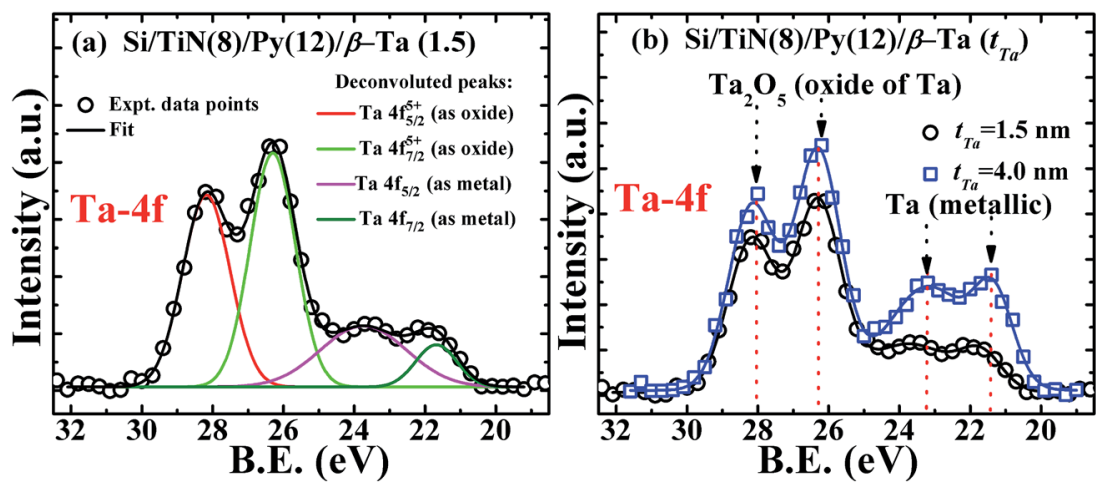

Fig. 8 (a) The XPS spectra of Ta- $4 \mathrm{f}$ for epi-Py $(12 \mathrm{~nm}) / T a(1.5 \mathrm{~nm})$ bilayer showing $4 \mathrm{f}_{5 / 2}$ and $4 \mathrm{f}_{7 / 2}$ peaks corresponding to oxidized and metallic tantalum. Lines represent the de-convoluted peaks. (b) Comparison of the XPS spectra of epi-Py (12 nm)/Ta (1.5 nm) bilayer with the epi-Py (12 $\mathrm{nm}) / \mathrm{Ta}(4 \mathrm{~nm})$ bilayer.

By the time Ta layer thickness reaches $6 \mathrm{~nm} \alpha$ has decreased to 0.0077 from 0.0087 for bare Py (i.e. now $-\Delta \alpha=0.0010$ ). As explained above, this progressive anti-damping behavior observed as $t_{\mathrm{Ta}}$ is increased beyond $1.5 \mathrm{~nm}$ to $6 \mathrm{~nm}$, attributed to Rashba effect in which anti-damping is a consequence of passing a DC-current through FM/NM bilayers that consists of $\mathrm{NM}$ layer lacking inversion symmetry. Below $t_{\mathrm{Ta}}=2 \mathrm{~nm}$, the reduction in damping, compared to that observed in case of bare Py layer, could also be in part due to the absence of possible oxidation of Py layer. ${ }^{26,58}$

Above discussion satisfactorily accounts for the observed anomalous decrease in $\alpha_{\text {eff }}$ in the present case of Py/ $\beta$-Ta, we however do not rule out any other plausible physical process accounting for the observed decrease in $\alpha_{\text {eff }}$ with the increase in thickness in the $t_{\mathrm{Ta}}<2 \lambda_{\mathrm{SD}}$ regime. The present results therefore suggest the need for further comprehensive modeling of the lower thickness regime of nonmagnetic metal. Having obtained the minimum $\alpha_{\text {eff }}$ of 0.0077 in Py $(12 \mathrm{~nm}) / \beta$-Ta $(6 \mathrm{~nm})$ bilayers consisting of 2-D Py layer having uniform magnetization precession (minimum TMS) and $\sim 2 \lambda_{\mathrm{SD}}$ thick $\beta$-Ta cap layer, we were curious to see what will be the $\alpha_{\text {eff }}$ if 3-D Py is used in the bilayers. Therefore we fabricated Py $(7 \mathrm{~nm}) / \beta$-Ta $(6 \mathrm{~nm})$ bilayers consisting of 3-D Py layer having significant non-uniform magnetization precession with optimal $\sim 2 \lambda_{\text {SD }}$ thick $\beta$-Ta cap layer and found an $\alpha_{\text {eff }}$ of 0.0090 , which is nearly the same as that for the bare Py layer. Similar situation of high damping constant of bare Py layer and no decrease on adding optimal $\beta$ Ta cap layer was also observed for 3 and $5 \mathrm{~nm}$ thick 3-D Py layers. This absence of anti-damping behavior in non-uniformly precessing Py layers $\left(t_{\mathrm{Ta}}<10 \mathrm{~nm}\right)$ on adding optimal $(\sim 6 \mathrm{~nm}) \beta$ Ta cap layer is due to diminishing of net non-equilibrium induced anti-damping torque or might be linked to the spin wave induced spin pumping effect. ${ }^{59}$

\section{Conclusions}

We have prepared epitaxial Py layers on TiN buffered Si and have studied the correlation of epi-Py layer thickness with the extrinsic TMS contribution to FMR line-width $\Delta H$ and associated Gilbert damping constant $\alpha$. A non-linear variation of $H_{\mathrm{r}}$ and $\Delta H$ with $t_{\mathrm{Py}}$ is found for $t_{\mathrm{Py}}<12 \mathrm{~nm}$ indicating the dominant nature of non-uniform magnetization precession mode responsible for generating the extrinsic TMS. On the other hand, above $\geq 12 \mathrm{~nm}$ of $t_{\mathrm{Py}}$, the $H_{\mathrm{r}}$ and $\Delta H$ become constant and independent of Py layer thickness in the studied thickness range of 12-20 $\mathrm{nm}$. This confirms the dominant presence of uniform magnetization precession mode and significant reduction in extrinsic TMS contribution. We have also observed the effect of change in the thickness of the $\beta$-Ta layer deposited over the optimized 2-D epitaxial Py $(12 \mathrm{~nm})$ layer on the intrinsic spin-orbit contribution in terms of changes in the $\Delta H$ and $\alpha_{\text {eff. }}$ We found that in the presence of $\beta$-Ta layer of thickness less than $\sim 6 \mathrm{~nm}$, the effect of anti-damping spin-orbit torque acts on the uniform magnetization precession in Py layer and results in anomalous reduction in the $\Delta H$ and $\alpha_{\text {eff }}$ in comparison to bare Py (12 nm) layer. An attempt has been made to account for the observed effect of change in the $\beta$-Ta layer thickness on the anti-damping spin-orbit torque based on the interfacial spin accumulation concept and absorption of spin angular momentum at the FM/NM interface. The $t_{\mathrm{Ta}} \approx 6 \mathrm{~nm}\left(2 \lambda_{\mathrm{SD}}+2\right.$ $\mathrm{nm}$ ) is found to be optimum thickness of $\beta$-Ta over epitaxial Py film possessing uniform magnetization precession, that is useful for STT device applications owing to maximum transfer of spin current.

\section{Acknowledgements}

Useful discussions with Dr P. K. Muduli are thankfully acknowledged. The FIST-DST, Govt. of India is thankfully acknowledged for the XPS measurements at IIT Delhi. One of the contributing authors (N. B.) acknowledges MHRD, Govt. of India for providing Senior Research Fellowship.

\section{References}

1 C. Chappert and J. V. Kim, Nat. Phys., 2008, 4, 837-838.

2 K. Ando, Y. Kajiwara, S. Takahashi, S. Maekawa, K. Takemoto, M. Takatsu and E. Saitoh, Phys. Rev. B: Condens. Matter Mater. Phys., 2008, 78, 014413. 
3 O. Mosendz, J. E. Pearson, F. Y. Fradin, G. E. W. Bauer, S. D. Bader and A. Hoffmann, Phys. Rev. Lett., 2010, 104, 046601.

4 L. Liu, O. J. Lee, T. J. Gudmundsen, D. C. Ralph and R. A. Buhrman, Phys. Rev. Lett., 2012, 109, 096602.

5 L. Liu, C. F. Pai, Y. Li, H. W. Tseng, D. C. Ralph and R. A. Buhrman, Science, 2012, 336, 555-558.

6 L. Liu, T. Moriyama, D. C. Ralph and R. A. Buhrman, Phys. Rev. Lett., 2011, 106, 036601.

7 J. H. Park, C. H. Kim, H. W. Lee and J. H. Han, Phys. Rev. B: Condens. Matter Mater. Phys., 2013, 87, 041301.

8 T. D. Skinner, M. Wang, A. T. Hindmarch, A. W. Rushforth, A. C. Irvine, D. Heiss, H. Kurebayashi and A. J. Ferguson, Appl. Phys. Lett., 2016, 108, 121602.

9 A. R. Mellnik, J. S. Lee, A. Richardella, J. L. Grab, P. J. Mintun, M. H. Fischer, A. Vaezi, A. Manchon, E. A. Kim, N. Samarth and D. C. Ralph, Nature, 2014, 511, 449-451.

10 P. M. Haney, H. W. Lee, K. J. Lee, A. Manchon and M. D. Stiles, Phys. Rev. B: Condens. Matter Mater. Phys., 2013, 87, 174411.

11 G. Allen, S. Manipatruni, D. E. Nikonov, M. Doczy and I. A. Young, Phys. Rev. B: Condens. Matter Mater. Phys, 2015, 91, 144412.

12 X. Wang and A. Manchon, Phys. Rev. Lett., 2012, 108, 117201.

13 A. Manchon and S. Zhang, Phys. Rev. B: Condens. Matter Mater. Phys., 2009, 79, 094422.

14 J. C. Slonczewski, J. Magn. Magn. Mater., 1996, 159, L1-L7.

15 H. Kurebayashi, J. Sinova, D. Fang, A. C. Irvine, T. D. Skinner, J. Wunderlich, V. Novák, R. P. Campion, B. L. Gallagher, E. K. Vehstedt, L. P. Zârbo, K. Výborný, A. J. Ferguson and T. Jungwirth, Nat. Nanotechnol., 2014, 9, 211-217.

16 K. S. Ryu, L. Thomas, S. H. Yang and S. S. P. Parkin, Nat. Nanotechnol., 2013, 8, 527-533.

17 S. Emori, U. Bauer, S.-M. Ahn, E. Martinez and G. S. D. Beach, Nat. Mater., 2013, 12, 611.

18 V. E. Demidov, S. Urazhdin, H. Ulrichs, V. Tiberkevich, A. Slavin, D. Baither, G. Schmitz and S. O. Demokritov, Nat. Mater., 2012, 11, 1028-1031.

19 J. Kim, J. Sinha, M. Hayashi, M. Yamanouchi, S. Fukami, T. Suzuki, S. Mitani and H. Ohno, Nat. Mater., 2013, 12, 240-245.

20 S. Woo, M. Mann, A. Tan, L. Caretta and G. S. D. Beach, Appl. Phys. Lett., 2014, 105, 202404.

21 Y. Tserkovnyak, A. Brataas and G. E. W. Bauer, Phys. Rev. B: Condens. Matter Mater. Phys., 2002, 66, 224403.

22 A. Brataas, Y. Tserkovnyak, G. E. W. Bauer and B. I. Halperin, Phys. Rev. B: Condens. Matter Mater. Phys., 2002, 66, 060404(R).

23 H. Jiao and G. E. W. Bauer, Phys. Rev. Lett., 2013, 110, 217602. 24 Y. Tserkovnyak, A. Brataas, G. E. W. Bauer and B. I. Halperin, Rev. Mod. Phys., 2005, 77, 1375-1421.

25 A. Brataas, Y. Tserkovnyak, G. E. W. Bauer and P. J. Kelly, Spin pumping and spin transfer, published in "Spin Current", ed. S. Maekawa, E. Saitoh, S. Valenzuela and Y. Kimura, Oxford University Press, 2012, ch. 8.

26 N. Behera, S. Chaudhary and D. K. Pandya, Sci. Rep., 2016, 6, 19488.
27 S. Kim, D. J. Kim, M. S. Seo, B. G. Park and S. Y. Park, Appl. Phys. Lett., 2015, 106, 032409.

28 I. M. Miron, G. Gaudin, S. Auffret, B. Rodmacq, A. Schuhl, S. Pizzini, J. Vogel and P. Gambardella, Nat. Mater., 2010, 9, 230-234.

29 G. Tatara, N. Nakabayashi and K. J. Lee, Phys. Rev. B: Condens. Matter Mater. Phys., 2003, 87, 054403.

30 M. D. Stiles and A. Zangwill, Phys. Rev. B: Condens. Matter Mater. Phys., 2002, 66, 14407.

31 M. J. Hurben and C. E. Patton, J. Appl. Phys., 1998, 83, 43444365.

32 R. Arias and D. Mills, Phys. Rev. B: Condens. Matter Mater. Phys., 1999, 60, 7395-7409.

33 B. Heinrich and J. A. C. Bland, Ultrathin Magnetic Structures II: Measurement Techniques and Novel Magnetic Properties, 1994.

34 J. Narayan and B. C. Larson, J. Appl. Phys., 2003, 93, 278-285. 35 A. Kumar, D. K. Pandya and S. Chaudhary, Appl. Phys. Lett., 2013, 102, 152406.

36 H. An, Y. Kanno, T. Tashiro, Y. Nakamura, J. Shi and K. Ando, Appl. Phys. Lett., 2016, 108, 121602.

37 C. Kittel, Phys. Rev., 1948, 73, 155-161.

38 J. Okabayashi, J. W. Koo, H. Sukegawa, S. Mitani, Y. Takagi and T. Yokoyama, Appl. Phys. Lett., 2014, 105, 122408.

39 H. Lee, L. Wen, M. Pathak, P. Janssen, P. LeClair, C. Alexander, C. K. A. Mewes and T. Mewes, J. Phys. D: Appl. Phys., 2008, 41, 215001.

40 A. Azevedo, A. B. Oliveira, F. M. De Aguiar and S. M. Rezende, Phys. Rev. B: Condens. Matter Mater. Phys., 2000, 62, 53315333.

41 Z. O. Lei, G. J. Li, W. F. Egelhoff, P. T. Lai and P. W. T. Pong, IEEE Trans. Magn., 2011, 47, 602.

42 Z. O. Lei, G. J. Li, W. F. Egelhoff, P. T. Lai and P. W. T. Pong, IEEE Trans. Magn., 2011, 47, 714.

43 G. Li, C. W. Leung, C. Shueh, H. F. Hsu, H. R. Huang, K. W. Lin, P. T. Lai and P. W. Pong, Surf. Coat. Technol., 2013, 228, S437-S441.

44 G. Woltersdorf, "Spin-pumping and two-magnon scattering in magnetic multilayers", Ph.D. dissertation, Simon Fraser University, 2004, p. 22.

45 W. Alayo, S. Landi Jr, F. Pelegrini and E. Baggio-Saitovitch, J. Magn. Magn. Mater., 2014, 350, 100-106.

46 R. C. Oliveira, R. L. Rodriguez-Suárez, F. M. deAguiar, S. M. Rezende, J. R. Fermin and A. Azevedo, J. Magn. Magn. Mater., 2004, 272, e795-e796.

47 M. H. Read and C. Altman, Appl. Phys. Lett., 1965, 7, 51-52. 48 Y. Sun, H. Chang, M. Kabatek, Y. Y. Song, Z. Wang, M. Jantz, W. Schneider, M. Wu, E. Montoya, B. Kardasz, B. Heinrich, S. G. E. T. Velthuis, H. Schultheiss and A. Hoffmann, Phys. Rev. Lett., 2013, 111, 106601.

49 Y. Yang, Y. Wu, B. Wu, K. Yao, S. Shannigrahi and B. Zong, J. Appl. Phys., 2014, 115, 17 C509.

50 G. Woltersdorf, O. Mosendz, B. Heinrich and C. H. Back, Phys. Rev. Lett., 2007, 99, 227207.

51 A. Brataas, Y. V. Nazarov and G. E. W. Bauer, Phys. Rev. Lett., 2000, 84, 2481-2484. 
52 A. A. Abrikosov and L. P. Gor'kov, Sov. Phys., 1962, 15, 752757.

53 R. Meservey and P. M. Tedrow, Phys. Rev. Lett., 1978, 41, 805808.

54 Q. Yang, P. Holody, S. F. Lee, L. L. Henry, R. Loloee, P. A. Schroeder, W. P. Pratt and J. Bass, Phys. Rev. Lett., 1994, 72, 3274-3277.

55 H. Nakayama, K. Ando, K. Harii, T. Yoshino, R. Takahashi, Y. Kajiwara, K. Uchida, Y. Fujikawa and E. Saitoh, Phys. Rev. B: Condens. Matter Mater. Phys., 2012, 85, 144408.
56 X. Fan, H. Celik, J. Wu, C. Ni, K. J. Lee, V. O. Lorenz and J. Q. Xiao, Nat. Commun., 2014, 5, 3042.

57 H. Zhao, G. Yu and H. Si, J. Mater. Sci. Technol., 2004, 20, 239-240.

58 A. Ruiz-Calaforra, T. Brächer, V. Lauer, P. Pirro, B. Heinz, M. Geilen, A. V. Chumak, A. Conca, B. Leven and B. Hillebrands, J. Appl. Phys., 2015, 117, 163901.

59 J. H. Kwon, J. Yoon, M. Hayashi, P. Deorani, H. Yang, J. M. Lee, J. Sinha and K. J. Lee, Sci. Adv., 2016, 2, e1501892. 